\title{
Polysaccharide-based scaffold for tissue-regeneration
}

\author{
Stefano Rimondo*, Giuseppe Perale ${ }^{*}$, Filippo Rossi* \\ *Dipartimento di Chimica, Materiali e Ingegneria Chimica "Giulio Natta," \\ Politecnico di Milano, Milan, Italy, †Biomaterials Laboratory, Institute for \\ Mechanical Engineering and Materials Technology, University of Applied Sciences \\ and Arts of Southern Switzerland, Manno, Switzerland
}

\subsection{Introduction}

In the last 10 years, the field of transplantation has dramatically improved, increasing patient life expectations and graft survival, but the demand for tissue and organs continues to exceed supply. Currently, every $10 \mathrm{~min}$, someone is added to the national transplant waiting list (United States) that consists of 115,000 people, but only $30 \%$ can be treated [1]. The synergy between regenerative medicine and tissue engineering represents an innovative strategy to lighten this demand. The focus of regenerative medicine is the body's intrinsic tissue repair, but that is not enough. Tissue engineering was introduced by Vacanti and Langer in the 1980s; and the widespread belief is represented as the well-known image of the "human ear on the mouse back." The goal of this modern technology is to create a substitute tissue, which supplies an individual therapy for patients with regeneration, remodeling, and growth potential [2]. That is possible thanks to three fundamental components: cells, biomaterial scaffold, and signals (Fig. 6.1).

The classic paradigm relies on a combination of these three factors to orchestrate tissue formation and integration within the host environment. An important avenue of tissue engineering is the development of biomaterials that can promote regenerative processes by effectively transporting cell populations and therapeutic agents, as well as providing structural scaffolding that confers sufficient mechanical properties to tissues. Moreover, the biomaterial should ideally degrade at a comparable rate to growth of new tissue at the site of implantation [3].

Focusing on biomaterials, the polysaccharides are valid candidates to synthesize scaffold for cell supporting (e.g., three-dimensional hydrogels), drug delivery (e.g., microgels/nanogels), and self-assembly micelles for gene therapy. Polysaccharides are carbohydrates whose molecules consist in a number of sugar moieties linked together and are abundant in nature, renewable, nontoxic, and intrinsically biodegradable. They are naturally occurring polymers or biopolymers. Typical examples of polysaccharides include: dextran (DeX), starch (AmY), glycogen (Gly), cellulose (CeL), chitosan (CS), hyaluronic acid (HA), alginate (ALG), and many others [4]. 


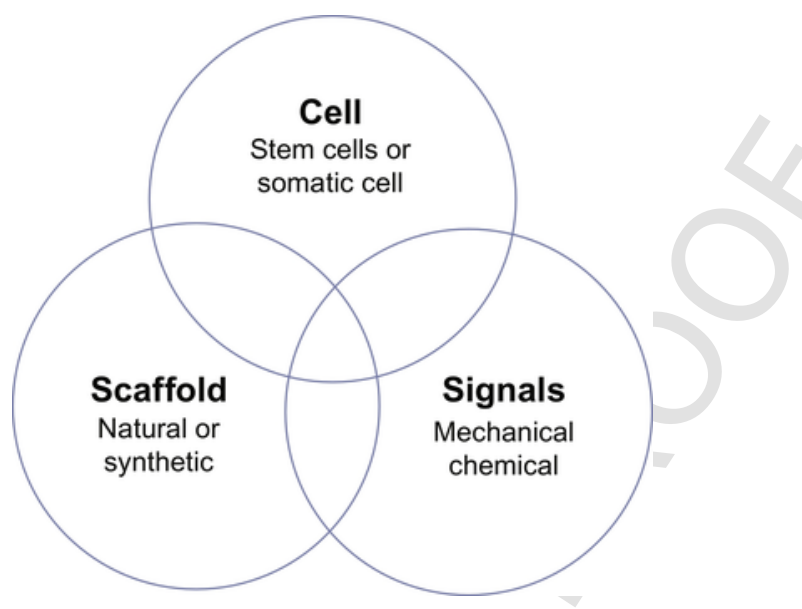

Fig. 6.1 Fundamental components of tissue engineering.

They are characterized by hydroxyl, amino, and carboxyl groups that could provide specific functionality and biological recognition features or serve to tune biological or mechanical properties of the parent molecules. As indicated by the European Polysaccharide Network of Excellence (EPNOE), research on polysaccharides has attracted tremendous academic interest and fundamental understanding that will boost the development of applications, such as in this case for medical application [5]. Most polysaccharide-based biomaterials are still used in preclinical studies, and the interest is increasing due to their hydrophobic nature that makes them good candidates for hydrogel synthesis. Currently, this biomaterial is widely used in a lot of body systems like nervous, musculoskeletal, digestive, respiratory, urinary, circulatory, and integumentary system. This chapter focuses on the principal characteristics and uses of the common polysaccharide in tissue regeneration.

\subsection{Principles of tissue regeneration}

As previously said, regenerative medicine is based on the body's intrinsic tissue repair that occurs following three phases: the hemorrhage and clot formation, granulation tissue formation, and contraction. The first phase is characterized by blood escaping from the circulatory system followed by an activation of platelets and release of coagulation factor to make a matrix called a "clot." This allows the extravasation of cells from the immune system. In the second phase, tissue cells called macrophages migrate toward the damaged area and release factors that recall fibroblasts and endothelial cells; these cells in turn form the granulation tissue. Fibroblasts are the protagonists of the last phase with the secretion of collagen and dedifferentiation into myofibroblasts, inducing tissue contraction and remodeling. 
As we see, cells that are the building blocks of tissue play a key role in the regeneration of tissues that themselves are the basic unit of the body. Generally, groups of cells make and secrete their own support structures, which is called an extra-cellular matrix (ECM). This matrix, or natural scaffold, is very important not only for supporting cell growth but also because it acts as a relay station for various signaling molecules [6]. The process often begins obtaining biological material (as entire organs or tissue, umbilical cord, and placenta) from a donor that can be alive or dead. (There is a possibility that the donor is an animal.) Then there is a purification, isolation, and expansion process in order to obtain the desired cells culture.

After that, there is the building scaffold phase previously designed from a wide set of possible sources (natural or synthetic scaffold). Once scaffolds are synthesized, cells can be introduced on it with or without a "cocktail" of growth factor. In some cases, the cells, scaffolds, and growth factors are all mixed together at once, allowing the tissue to "self-assemble." Another method to create new tissue is represented by the use of an existing scaffold with decellularized tissue and organs. The cells of a donor organ are stripped, and the remaining decellularized scaffold is used to grow new tissue. This process has been used to bioengineer liver, lung, heart, and kidney tissue. This approach holds great promise for using scaffolding from human tissue discarded during surgery and combining it with a patient's own cells to make customized organs that would not be rejected by the immune system. When we consider the application of tissue regeneration (that is strictly related to tissue engineering), it is widely used as a general process that can be summarized as follows (Fig. 6.2).

\subsection{Polysaccharide characteristics and properties}

Polysaccharides are subclass organic compounds of carbohydrates whose molecules consist in a number of sugar moieties bound together by glycosidic linkages in order to make big, complex molecules. Some structural features are exhibited in natural occurring polysaccharides as molecular weight, monosaccharide and disaccharide composition, glycosidic linkage patterns with two types of configuration $\alpha$ or $\beta$, degree of branching, charging properties, etc. All of these structural properties determine the functional properties, such as solubility. Understanding solubility of polysaccharides is fundamental in that most polysaccharides perform their function in aqueous system. Polysaccharides show a range of solubility indeed; some are water insoluble, such as cellulose, and others are only hot water soluble, such as starch. Still others can be readily dissolved in cold water, such as pullulan and gum arabic [7]. Usually polysaccharides contain small amounts of sugar derivative; examples are: esters of phosphoric acid (phosphates), sulfuric acid (sulfates), and acetic acid (acetates).

Polysaccharides can be classified in different ways, such as on the basis of structure, chemical composition, physical properties, sources, and applications. Regarding chemical composition, polysaccharides are divided into two broad 


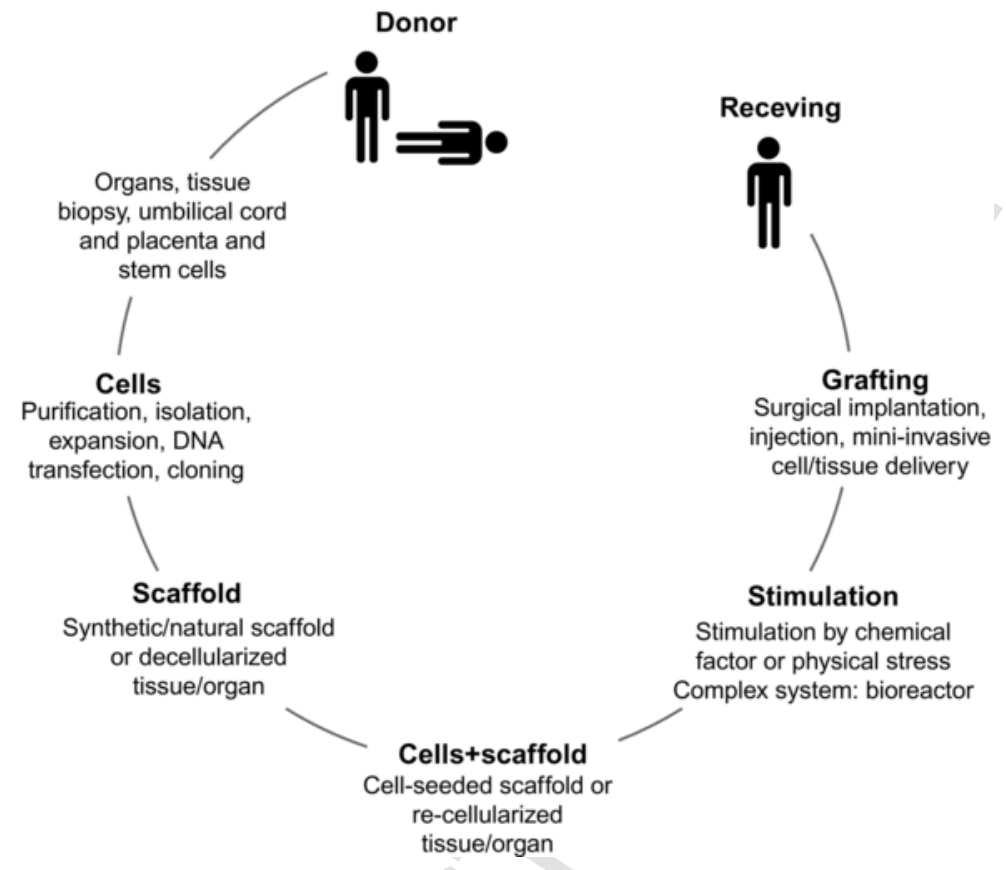

Fig. 6.2 Tissue regeneration general process.

classes: homoglycans and heteroglycans [8]. Respectively, we find polysaccharides as cellulose, starch, glycogen in homoglycans class, polysaccharides as chitosan, alginate, glycosaminoglycans (GAGs), and dextran in heteroglycans class. Homoglycans or homo-polysaccharides are a class of polysaccharides formed by the same ("homo-") kind of monosaccharide while heteroglycans or hetero-polysaccharides are a class of polysaccharides formed by two or more different ("hetero-") kind of monosaccharides (repetition of a disaccharide unit). Monosaccharide and disaccharide structures found most commonly in polysaccharide are respectively: D-glucose, D-mannose, D-galactose, 2-acetamido-2-deoxy-D-glucose, L-arabinose, D-xylose, L-rhamnose, 2-amino-2-deoxy-D-glucose, L-iduronic acid, D-glucuronic acid, D-galacturonic acid for monosaccharide side and for disaccharide side found lactose, maltose (reducing disaccharide) and saccharose, $\alpha, \alpha$-trehalose (nonreducing disaccharide). Polysaccharides play two important roles in organisms: first, they are used as energy storage; second, they have structural and protection function. Currently, the interest in polysaccharide-based biomaterials is increasing. Three aspects have contributed to this increase; first is the expanded knowledge on the molecular and cellular basis of health and disease, human and animal bodies, and the role of saccharide moieties in cell signaling/recognition scheme. Second is the development of new synthetic techniques for design active oligosaccharide for biological application and techniques to study the oligosaccharide signaling mechanisms The last factor is the introduction of novel 
materials with specific characteristics for biological application (biocompatibility, biodegradability, nontoxicity drug releasing, etc.) due to the great expansion of tissue engineering in research panorama [9]. One of the important properties of polysaccharides in general is their ability to form hydrogels. Currently, hydrogels of these polymers are being widely studied in biomedical areas due to their excellent biocompatibility, biodegradability, nontoxicity, drug-releasing properties, etc. Thanks to their ability to swell and shrink and to make hydrogels from such polysaccharides as smart carrier devices has greatly revolutionized these materials in drug delivery and/or drug targeting areas [10]. Polysaccharide gel formation is generally driven by physical interactions: hydrogen bonds and ionic interactions (Fig. 6.3).

\subsection{Homo-polysaccharide}

Homo-polysaccharides are a class of polysaccharides formed by the same ("homo-") kind of monosaccharide. The principal homo-polysaccharides are cellulose, starch, and glycogen. Each of them is composed by hundreds of molecules of glucose linked by glycosidic bonds.

\subsubsection{Cellulose (CeL)}

Cellulose is an organic homo-polysaccharide present principally in the vegetables as the major component of plant cell walls. Its linear structure is constituted of long chains of D-glucose subunits joined together by $\beta(1-4)$ glucosidic bond. These long

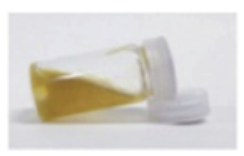

sol

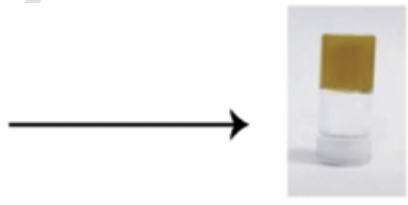

gel
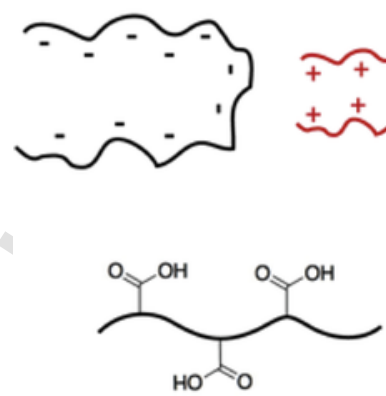
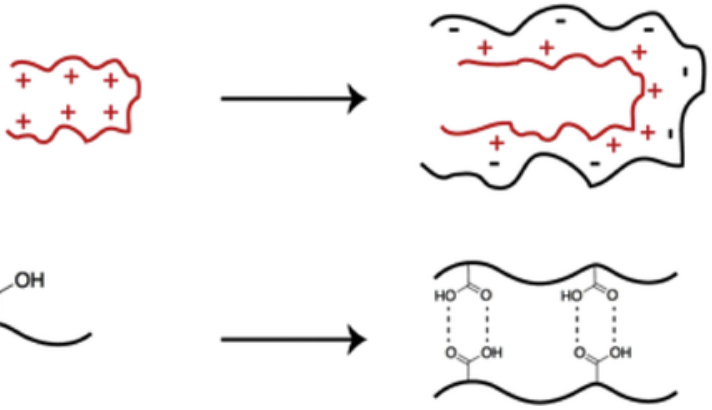

Fig. 6.3 Gel formation driven by physical interactions. 
chains are arranged parallel to each other and are joined together by hydrogen bonds to form microfibrils [11]. The aggregates of microfibrils make the fibrin of cellulose that in natural form is called Cellulose I. The cellulose is difficult to dissolve and digest because the $\beta(1-4)$ glucosidic bonds can only be broken-down by cellulase. This characteristic makes cellulose a candidate for biomaterial. Cellulose produced by bacteria, also known as "bacterial cellulose (BC)," is a material that is receiving increasing attention for its biological potential. Principally, BC is produced by some bacteria of the genera Acetobacter, Sarcina, Gluconacetobacter, or Agrobacterium [12]. The $\mathrm{BC}$ has several advantages compared to conventional vegetable cellulose, such as:

- Cellulose can be obtained at very high purity without lignin or other contaminants, so no energy is required for its purification as it is required for vegetable one;

- The possibility of having properties that do not depend on the climatic conditions of production; microorganisms can be genetically modified to produce cellulose with the desired properties;

- The growth rate of microorganisms can be controlled to produce cellulose in the required quantities and in the desired time interval;

- Agricultural and industrial waste can be used as a growth medium, so production is relatively cheap.

Currently, $\mathrm{BC}$ is used in different fields as alimentary (food functionalization), cosmetic (as an additive), and in the biomedical sector (as wound-dressing material, scaffolds in tissue-engineering, artificial skin, and advanced drug-delivery systems) [12-16].

$\mathrm{BC}$ is characterized by good properties in terms of mechanical properties (high tensile strength), physical characteristics (swelling behavior, high water absorption), porosity, ultra-fine fiber network, (mold-ability) and excellent biological affinity (biodegradability, nontoxicity, and biocompatibility) [17]. An interesting use of BC is related to bone-tissue engineering. Due to multiple conditions including traumatic events, tumors, and disease, the research on bone restoring/regeneration has great weight in clinical panorama. Ceramics, composite materials, metal, or synthetic polymers that have been examined as potential scaffolds show good grades of biocompatibility and degradability, but they have important drawbacks; they are brittle and have low mechanical stability [18]. Considering these facts, $\mathrm{BC}$ is an emerging biomaterial that is proposed as an alternative to the other materials covered.

Several studies in vivo and in vitro for evaluating $\mathrm{BC}$ as a suitable biocompatible candidate for bone tissue engineering was conducted by several groups. For bone application, a microscopic pore structure is needed to facilitate osteoblast ingrowth and formation of mineralized tissue. However, BC-nanofibrils form a dense mesh that can limit cell infiltration. So to improve a good environment for cells, porosity was introduced into the material by incorporating porogens into the fermentation process. The removal of porogens led to the formation of a $\mathrm{BC}$ network of interconnected pores. From literature, microporous network with pore-size of 300-500 $\mu \mathrm{m}$ provided a suitable environment for bone cells [19]. Zaborowska et al. 
have searched a method to form microporous 3D BC-scaffolds with adequate porosity. In this specific case, paraffin microspheres were incorporated in the fermentation process and subsequently removed. Scanning electron microscopy showed clearly the difference between a BC-scaffold and porous BC-scaffold. As you see in Fig. 6.4; regarding the mechanical properties, tensile measurements showed a Young's modulus of 1.6 MPa.

In order to prove that microporous $\mathrm{BC}$ is a promising biomaterial for bone tissue engineering applications, a cell study was performed.

Microporous $\mathrm{BC}$ and $\mathrm{BC}$ with nanopores (as control) were seeded with osteoprogenitor cells; then confocal microscopy and histology was performed to evaluate cell distribution and mineral deposition. Confocal microscopy has revealed that the seeded cells appear at confluence on both porous and control scaffolds, but BC-microporous presented a better cell infiltration with consequent dense clusterization. However, cells in microporous BC formed denser mineral deposits than cells grown on the nano-porous BC. In fact, in accordance with literature, mineral deposition is enhanced when osteoprogenitor cells are arranged in dense clusters as in the case of microporous-BC [20]. Due to this evidence, we can consider that bacterial cellulose is interesting as a future scaffold for bone regeneration.

The ease of manipulating to suit any desired direction by chemical reactions, structural changes, or inclusion of secondary components into the BC structure has made it as an ideal biomaterial for the study performed by Josè G.H. The BC-based scaffold involved in this study was proposed as a suitable 3D system for osteoblast cell culture. Even if BC presents good capacity for water absorption, porosity, stability, biocompatibility, and its mechanical properties, it is not optimal for bone regeneration if used alone. So, to overcome these limits and improve the mechanical
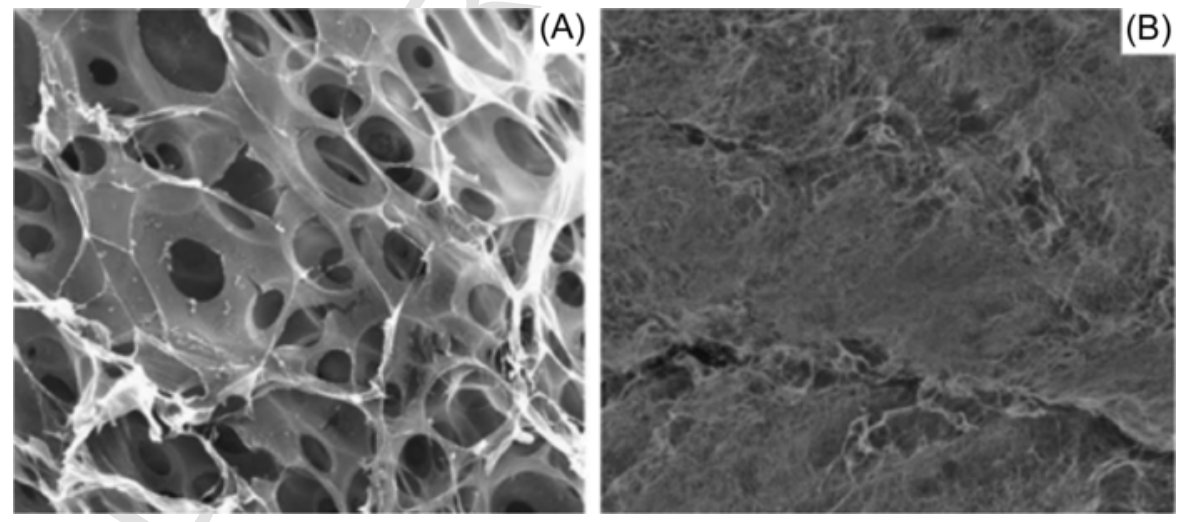

Fig. 6.4 SEM images. (A) The morphology of porous BC produced with paraffin particles of size 300-500 $\mu \mathrm{m}$. (B) The morphology of BC without micropores.

Reprinted with permission from Elsevier: Zaborowska M, Bodin A, Bäckdahl H, Popp J, Goldstein A, Gatenholm P. Microporous bacterial cellulose as a potential scaffold for bone regeneration. Acta Biomater 2010;6:2540-7. 
properties, $\mathrm{BC}$ was used in combination with functionalized multiwalled carbon nanotubes (MWNTs). In order to fabricate BC-MWNTs scaffold, pellicles of native $\mathrm{BC}$ were broken down by high-intensity mixing to obtain a fibrous pulp of $\mathrm{BC}$, whereas MWNTs-COOH powder was suspended in deionized water and sonicated to obtain MWNTs-COOH suspension.

These two components were mixed with ultrasonic tip and solution; then by adding alginate/D-mannitol and a cross-linking solution $\left(\mathrm{CaCl}_{2}\right)$, the scaffold was obtained. Rheology tests on this new scaffold shows that mechanical properties of native $\mathrm{BC}$ can be increased with the addition of carbon nanotubes; in particular, the storage modulus $\left(G^{\prime}\right)$ of BC/MWNTs-COOH scaffold is higher than native BC scaffold. The storage modulus increases with increasing concentration of carbon nanotubes as shown below (Fig. 6.5A).

BC-MWNTs-COOH was tested with osteoblast cell and cell viability; proliferation and adhesion were evaluated. Regarding cell viability and adhesion, the use of $\mathrm{BC}$ in combination with carbon nanotubes shows important levels of osteoblast viability and adhesion while the only use of $\mathrm{BC}$ presents a lower level of viability. Coherently with what has just been said, cell proliferation was evaluated, and it was once again observed that BC-MWNTs scaffolds show elevated levels of osteoblast proliferation (Fig. 6.5B). Due to these facts, BC, if used with carbon nanotubes, presents great potential for generation of bone tissue [21]. Another study concerning the use of $\mathrm{BC}$ as a bone regeneration material uses it in combination with type I collagen (COL1).
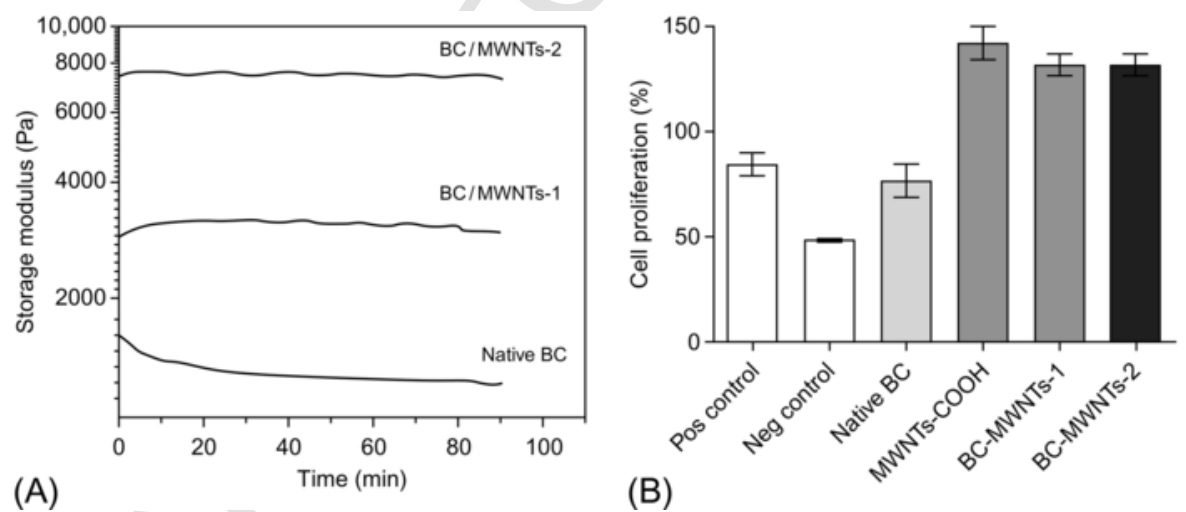

Fig. 6.5 Mechanical properties and cell proliferation. (A) Storage modulus of the following scaffold: BC, BC with low concentration of MWNTs-COOHs-1 $(0.25 \mathrm{mg} / \mathrm{mL})$ and BC with high concentration of MWNTs-COOHs-2 $(0.5 \mathrm{mg} / \mathrm{mL})$. (B) Cell (osteoblast) proliferation on different scaffold.

Reprinted with permission from Elsevier: Gutiérrez-Hernández JM, Escobar-García DM, Escalante A, Flores H, González FJ, Gatenholm P, Toriz G. In vitro evaluation of osteoblastic cells on bacterial cellulose modified with multi-walled carbon nanotubes as scaffold for bone regeneration. Mater Sci Eng C 2017;75:445-53. 
This polysaccharide was used in the form of highly hydrated $\mathrm{BC}$ membranes that were obtained from bacterial culture, and they are characterized by a 3D ultrafine matrix of cellulose nanofibers ("nanocelluloses"). Collagen represents $90 \%$ of the bone organic matrix, so its incorporation into different material (as in this case with BC) can fabricate bio-materials able to produce better tissue and cell responses, increasing cell adhesion and proliferation. The combination of bacterial cellulose membranes and type I collagen occurred by esterification of glycine and exploiting the 1-ethyl-3-(3-dimethylaminopropyl)-carbodiimide. Using X-rays, it is possible to observe that the addition of collagen modifies the crystallinity of BC. Regarding the mechanical properties of the BC-COL1 system, there is a lower elastic modulus and a lower tensile strength if compared to only the BC system. BC-COL1 was tested with osteogenic cells. Cell culture experiments revealed that cell morphology and cell viability/proliferation was not different between the cultures grown on $\mathrm{BC}$ or BC-COL1. However, after 17 days of cultured cells, BC-COL1 allowed the development of the osteoblastic phenotype in vitro. Thus, BC-COL should be considered as alternative biomaterial for bone tissue engineering [22]. In addition, Aase Bodin et al. studied how to produce 3D bacterial cellulose scaffold seeded with human urine-derived stem cells (USC) to create a tissue-engineered conduit for urinary reconstruction and diversion. The $\mathrm{BC}$ scaffold was prepared by adding sterile paraffin particles to a tubular fermentation vessel containing a type of Acetobacter [23] as shown in Fig. 6.6. After 7 days of bacterial culture, 3D BC-based network was obtained; then it was purified, cut into $5 \mathrm{~mm}$ thick sheets, and finally sterilized before the cell seeding. Urine stem cells (USC) were induced to differentiate into urothelial and smooth muscle cells (SMC). SMC cells were seeded onto synthetized BC scaffold under static and 3D dynamic conditions and cultured for 2 weeks.

SMC and urothelial cells formed multilayers on the BC scaffold surface, and some cells were observed inside the scaffold. In addition, an in vivo test was performed: USC-BC scaffold constructs were implanted into athymic mice, and the cells were tracked. The immunohistochemical evaluation showed that cells are able to differentiate and express urothelial and SMC markers, so under these preliminary results, porous $\mathrm{BC}$ scaffold promotes $3 \mathrm{D}$ growth and differentiation of urine stem cells. Cell-seeded bacterial cellulose scaffolds represent a promising regenerative engineering tool for urinary reconstruction [15].

Once the skin loses much of its integrity or is severely damaged, it is unable to regenerate itself. In these cases, the tissue engineering approach holds promise for treating such defects. Aysen Tezcaner's group developed 3D electrospun cellulose acetate pullulan scaffolds for skin tissue engineering application. Pullulan, a polysaccharide polymer consisting of malt-triose units, is used to obtain three-dimensional structure. Electrospinning is a simple and applicable method to process cellulose and produce porous and fibrous meshes mimicking the natural ECMs of tissues. Mechanical and biological properties were evaluated, and cell culture studies were performed using mouse fibroblastic cell line (L929). In vitro cell culture tests showed that cells can adhere, proliferate, and populate within CA/ PULL scaffold [24]. Minseong Kim and GeunHyung Kim developed another type of 


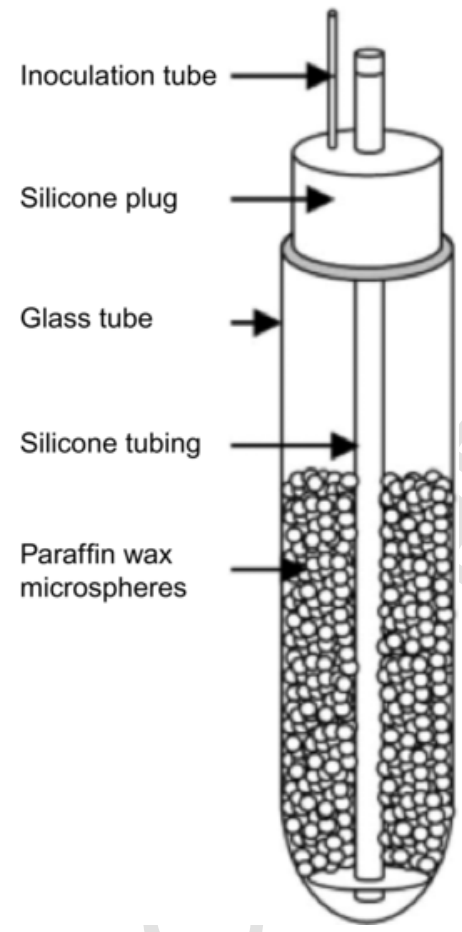

Fig. 6.6 Scheme of the fermentation set-up to prepare BC 3D scaffold. The Acetobacter culture produces an entangled BC network close to paraffin particles.

Reprinted with permission from Elsevier: Zaborowska M, Bodin A, Bäckdahl H, Popp J, Goldstein A, Gatenholm P. Microporous bacterial cellulose as a potential scaffold for bone regeneration. Acta Biomater 2010;6:2540-7.

scaffold consisting of multilayered fiber as potential tissue-regenerative material. It was fabricated by an electrohydrodynamic direct jet (EHDJ) process.

They compared the properties of the new scaffold with a synthetic PCL one with similar geometry and morphology. In vitro biocompatibility of both scaffolds were performed using human dermal fibroblasts. They observed that the cellulose structure showed higher cell adhesion and metabolic activities compared with the PCL ones. These results suggest that cellulose scaffold has high potential as a tissue regenerative material, and the EHDJ process is a valid technique for processing cellulose [25].

In addition, additive manufacturing (3D bioprinting technique) seems to be promising in the endeavor to create potentially replaceable cartilage tissue composed by human chondrocytes in combination with Nanocellulose-Alginate Bioink. The principal advantage of the 3D bioprinter is that with this technique, it is possible to create complex structure (as in physiological environments) from the bottom up, moving and dispensing material in each Cartesian direction. The printed structure 
created by this team-work is based on "bioink" (nanocellulose and alginate) mimic cartilage tissue with high fidelity and stability. Thanks to the good rheological properties, "bioink" also provided excellent printability with living cells. For these reasons, human chrondrocytes were used in this study and showed a cell viability of $73 \%$ after 1 day and $86 \%$ after 7 days. In view of these results, we can consider cellulose-based 3D material as a potential tool for living tissues and organ regeneration [26].

\subsubsection{Starch (AmY)}

Starch or amylum is a homo-polysaccharide produced by all vegetables as an energy store. Due to its presence in staple foods (bread, pasta, rice, potatoes, etc.), starch is the most common carbohydrate in human diets. The starch molecules $\left(\mathrm{C}_{6} \mathrm{H}_{10} \mathrm{O}_{5}\right)$ consist of a considerable number of glucose units joined by glycosidic bonds. It exists in two forms: the amylose and the amylopectin. The amylose is the linear and helical form composed by $\alpha(1-4)$ linkages. Branching takes place with a $\alpha(1-6)$ linkages. While amylopectin is the branched form, it has a structure like glycogen. Typically, pure starch is insoluble in cold water and some organic solvent (alcohol, ether, and acetone), but when it is heated up to a critical temperature called "gelatinization temperature," it becomes soluble in water in order to make stable gels. Synthetic starch can be used as carrier for various molecules in drug delivery due to its well-controlled degree of polymerization that makes it a suitable candidate for making nanoparticles for novel medical strategies.

Starch nanoparticles usually have been obtained by carboxylation and the oxidation process [27]. Drug delivery from starch-based nanoparticles to specific sites like colon, lungs, and cancer cells has been studied by several groups. Prajakta Dandekar et al. developed nanoparticles obtained from a hydrophobic propyl starch (using emulsification-diffusion technique) loaded with docetaxel (anticancer agent).

In their study, it is observable that nanoparticles with drugs are able to enhance internalization by Caco-2. NHDF-p cells are cancerous, and it was observed that their peri-nuclear localization confirmed the delivery of drugs [28]. Assam Bora rice, a variety of Indian rice, contains a type of starch that has been evaluated in vitro and vivo as a drug carrier in the form of bio-adhesive microsphere for colon-targeting by Ahamad team-work. A derivative of starch sold under the brand name of Voluven (Hydroxyethyl Starch) is used to prevent shock followed by surgery, trauma, and other accidents that generate a severe blood loss. It was demonstrated in the endotoxic rat model that Hydroxyethyl Starch has beneficial effects on capillary leak in acute lung injury [29], but in clinical treatment panorama, it presents some adverse effects including anaphylactoid reactions and renal failure. A scaffold based on a blend of corn starch/ethylene-vinyl alcohol (SEVA-C) was characterized in vitro to make a novel engineering material for the bone-tissue regeneration. Cytotoxicity evaluation was performed, and it revealed that the developed system is nontoxic and does not inhibit cell growth and proliferation. Direct contact assays were also carried out by use of a cell line of human osteoblast-like cells (SaOS-2). Cells were seeded and allowed to grow for 4 weeks in a controlled environment. Moreover, a cell 
viability assay (MTS test) demonstrated that cells were perfectly viable after the 4-week culture, showing the adequacy of the developed structure in supporting them. This good biological result shows that starch-based scaffolds should be considered as an alternative for bone tissue-engineering-regeneration applications in the near future [30].

\subsection{Hetero-polysaccharide}

Hetero-polysaccharides are a class of polysaccharides formed by two or more different ("hetero-") kind of monosaccharide (repetition of a disaccharide unit). The principal hetero-polysaccharides are chitosan, alginate, glycosaminoglycans (GAGs), and dextran. Each of them is composed by thousands of molecules. In nature, hetero-polysaccharides provide extracellular support for all the organisms. Together with fibrous proteins, including collagen, fibronectin, laminin, entactins, and others, they are considered the principal component of the extracellular matrix.

\subsubsection{Chitosan (CS)}

Chitosan is a linear amino-polysaccharide, composed of randomly distributed D-glucosamine and $N$-acetyl-D-glucosamine units linked by $\beta(1-4)$ glycosidic bonds formed by $N$-deacetylation of chitin [31].

Chitin, discovered by Henri Braconnot in 1811, is an unbranched polysaccharide (glucose-based) broadly distributed in nature as the principal component of cell walls in fungi, exoskeletons of crustaceans, insects, arthropods, and other invertebrates [32]. The two biopolymers that composed chitosan (D-glucosamine and $\mathrm{N}$-acetyl-D-glucosamine) are distinguishable from each other mainly by their solubility in acidic aqueous solutions [33]. When the degree of deacetylation of chitin reaches about $50 \%$, it becomes soluble in aqueous acidic media and is how the chitosan becomes one [34]. The interesting characteristics of chitosan, such as its physiochemical properties (molecular weight, degree of deacetylation, and crystallinity index) and its "bio-properties" (biocompatibility, nontoxicity, low allergenicity, antitumoral and antiviral activity, and biodegradability) allow it to be used in various medical and pharmaceutical applications [35]. As previously mentioned at neutral and alkaline $\mathrm{pH}$ and in some organic solvents, chitosan is insoluble, but in the presence of organic and inorganic acids, it forms water-soluble salts. In this environment, the amino groups of this hetero-polysaccharide become protonated and provide the molecule with positive charge. It is fundamental to consider that the portion on $\mathrm{N}$-acetyl-D-glucosamine units with respect to the total number of units present in the polysaccharide define as degree of acetylation influence the physicochemical properties [36]. The primary amino groups on chitosan can be functionalized; for example, the effect of the addition of a side chain is the increment of the amorphous fraction against a reduction of crystal structure. In base, on the nature of the side chain, the physiochemical properties change. 
Under mild conditions, the $\mathrm{pH}$-dependent (high-pH solution) solubility of chitosan represents a good property to create viscous solutions that can be gelled and extruded as fibers. These fibers can be, subsequently, high-strength fibers in dried form. For this reason, chitosan has been used for a lot of industrial processes based on film and fiber. Due to its cationic nature and high charge density in solution, chitosan is a suitable candidate for biomaterial. Another of chitosan's most promising features is its ability in porous structures (e.g., hydrogels) for use in cell transplantation and tissue regeneration [37]. Porous chitosan-based-scaffold can be obtained by freezing (controlling thermal gradients in order to obtain pore orientation) and then lyophilizing chitosan acetic acid solution in apposite stamp [38].

Different tissue engineering studies in which chitosan plays a key role are becoming increasingly numerous. CS scaffold is widely used in skin, bone, and cartilage healing. It has been used in regenerative medicine in two forms: 2D-scaffold and 3D-scaffold. The first one is principally related to dress wounds with film or membrane with controlled porosity, while the second one provides for a more $3 \mathrm{D}$ complex system in tissue engineering. A 3D-structure with controlled porosity presents considerable advantages, such as increased deposition of the matrix, which promotes regeneration, greater transport of nutrients, and removal of waste products. In order to obtain these 3D chitosan-based scaffolds, various team-works formulated chitosan as hydrogels, sponges, or foams. Chitosan is absent in mammals; nevertheless, when CS is put inside mammals, it disappeared rapidly or slowly depending on the degree of acetylation (DA), molecular weight, crystallinity, water percentage, and how the material is designed. The entire mechanism, however, is not totally clear; it is attributed to the presence of oxidative factor synthetized by immune system [39]. Today, there is a lot of attention on the regeneration of the skin following third-degree burns, abrasions, and other traumatic events that cause damage. Unfortunately, current treatments are still unsatisfactory, and, therefore many working groups, thanks to tissue engineering, propose alternative solutions. The ideal engineered biomaterial used for the regeneration of skin tissue should possess the following characteristics: microstructure similar to native tissue with average pore size of 100-200 nm, porosity over $90 \%$, excellent biocompatibility and biodegradability, adequate elastic properties [40]. Some examples of scaffolds based on chitosan for skin-tissue engineering are discussed. An example of CS-based scaffold application for skin repair is visible in the study conducted by Boucard et al. where they propose a new acellular biomaterial based on physical CS-hydrogel for treating severely burned skin. CS scaffold produced from squid pens was purified, dissolved in acetic acid, subsequently filtered on membranes, and, finally, after several washings, is freeze-dried. In order to mimic the skin, they proposed a hydrogel constituted of two physically linked layers where each one presents different mechanical properties and density. At first, rigid gel-layer (L1) was generated by evaporation of an hydroalcholic CS solution, while a second soft gel-layer (L2) was created by putting ammonia gas in contact with a CS solution [41] (Fig. 6.7). At the end, the entire gel was washed with water to eliminate the waste 


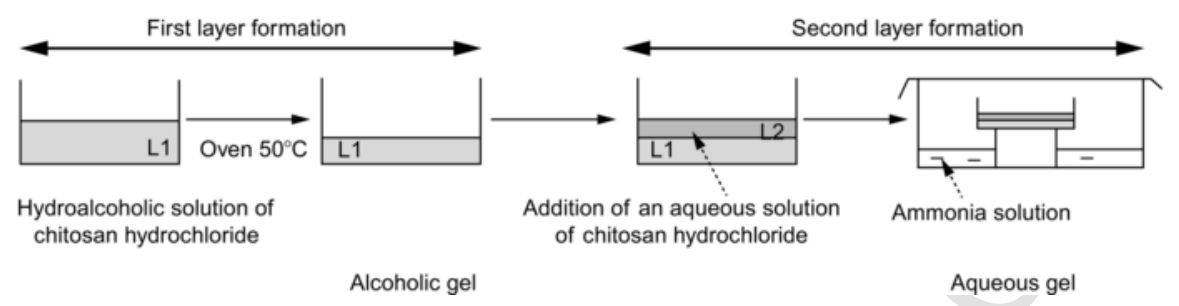

Fig. 6.7 Preparation of double-layer chitosan hydrogel.

Reprinted with permission from Elsevier: Boucard N, Viton C, Agay D, Mari E, Roger T, Chancerelle Y, Domard A. The use of physical hydrogels of chitosan for skin regeneration following third-degree burns. Biomaterials 2007;28:3478-88.

compound present during the synthesis. So the final double layer is composed only by chitosan and water [42].

CS-L1 is the rigid protective layer that has good mechanical properties and allows gas exchanges. CS-L2 instead is the second soft and flexible layer that allows the material to fit the geometry of the wound and ensure a good surface contact. To validate the biocompatibility of this CS-based biomaterial and its efficacy to improve the scar quality after burn injuries, two different experiments, E1 and E2, were performed. In the first one, four wounds were induced on the back of some pigs (two in the thoracic area and two in the lumbar area). The biopsies were performed at days 9 and 16 in order to evaluate the short-term tolerance. In the second one, the long-term tolerance was evaluated. E2 was performed as E1, but the biopsies were performed at days 100 and 293. The analysis showed that CS scaffolds were well tolerated and induced a good response for the regeneration of a limited portion of third-degree burned skin. This is due to its capability to induce inflammatory cell migration and high vascularization performed by angiogenetic properties. In E1 biopsy, it was observed that at day 22, a dermal-epidermal junction formed with the presence of collagens synthetized under the granulation tissue. In E2 at day 100, the new tissue obtained thanks to their CS bi-layer physical hydrogel mimicked quite well, in terms of aesthetic and flexibility, the native skin [42]. Other studies involved the use a CS-base biomaterial in testing for skin/wound healing. Hemcon Bandage (HCB) is a commercial chitosan-film made with CS acetate used for wounds closure. HCB and other similar chitosan-films are made by wet casting from CS salt solutions that are dried by oven or IR (infra-red). $\mathrm{HCB}$ is used for wounds closure as externally percutaneous arterial closure device (PACD). This system, even if it shows good efficacy, presents a deposition risk of harmful foreign bodies. HCB, in respect to standard manual compression (SMC), presents a positive efficacy and fast healing. Hemcon dressing also shows a good antibacterial property [43]. Ma et al. proposed 3D collagen/chitosan porous scaffolds for skin-tissue engineering. This scaffold provides an analogous structure to the extracellular matrix (ECM), ensuring adequate support and providing an environment necessary for cell infiltration, proliferation, and differentiation [9]. 
The technique used to fabricate it involved freeze-drying the mixture of collagen and CS solutions. Collagen and CS was dissolved separately in acetic acid solution; then the CS solution was slowly dropped into collagen suspension in the ratio of 9:1 (collagen:chitosan) and homogenized to obtain collagen/CS mixture. This mix was injected into a mold; then frozen and lyophilized for $24 \mathrm{~h}$ to obtain a porous 3D scaffold. In order to improve the biostability, the scaffold was treated with different concentrations of glutaraldehyde (GA). Confirmed by confocal microscopy, the GA concentration changes the cross-section morphology and the swelling ratios of synthetized scaffold. In vitro culture of human dermal fibroblasts confirmed that this GA-treated collagen/CS scaffold showed good cytocompatibility and accelerated cell infiltration and proliferation. Thanks to the good in vitro results in vivo animal tests were performed. The result underlined that the scaffold could support and accelerate the fibroblasts infiltration from the surrounding tissue, improving the skin regeneration and that the biodegradation of the scaffold is a long-term process. All these results suggest that collagen/chitosan scaffold cross-linked by GA is a potential candidate for dermal equivalent with enhanced biostability and good biocompatibility [44]. Another team focused on preparation and evaluation of novel chitosan biocompatible electronic skin. Chitosan electronic skin was prepared by a thermal-induced phase separation method, following treatment with $\mathrm{NaOH}$ gelating agent, followed by physical-chemical characterization (surface morphology, swelling behavior, mechanical strength, degradation rate). The electrode was produced with a screen-printing technique, followed by an adhesion analysis between the ink and cell biocompatibility. From scanning electron microscope (SEM) observations, $\mathrm{NaOH}$ gelated chitosan skin had the smooth surface morphology. The swelling ratio of all chitosan skin gelated by $\mathrm{NaOH}$ was less than $5 \%$. The chitosan skin, prepared by $300 \mathrm{kDa}$ chitosan gelated by $\mathrm{NaOH}$ for $3 \mathrm{~h}$, had the smallest swelling ratio. Furthermore, its mechanical strength showed the highest value of Young's modulus $(\sim 151 \mathrm{kPa})$. It indicated that $300 \mathrm{kDa}$ chitosan skins can produce hydrogels using $\mathrm{NaOH}$, and they present better size stability. All chitosan skins degraded about $20 \%$ of initial weight after 40 days in vitro. The adhesion strength of the silver inks on the surface of chitosan was better than carbon ink, and the electrical characteristic is almost the same with commercial polycarbonate (PC) substrate. MTT (3-(4,5-dimethylthiazol-2-yl)-2,5-diphenyl tetrazolium bromide) assay and SEM observation indicated that chitosan skin with silver electrode shows cell biocompatibility.

By further increasing the application of both screen imprinting technique and biopolymer, the idea of interdisciplinary collaborations can be achieved, and it would be a creative revolution to introduce the field of wearable devices into tissue engineering. Another area in which chitosan-based biomaterials are growing is represented by bone regeneration after trauma from accidental events (road accidents, falls, violence, etc.), resection of tumors, pathological fractures, bone infections, etc. [45]. Currently, some donor grafts (autografts, allografts, and xenografts) are used to solve some of these problems, but they present multiple limitations: for autografting. There is high morbidity rate and pain; while for allografts and xenografts, there is the risk of disease transmission and the high rate of 
rejection (immunogenicity). Precisely for these reasons, the interest on engineering biomaterials is growing considerably [45]. Recent studies have shown that CS presents chemical and biological properties similar to natural bone tissue. The application of CS-base scaffolds is interesting; they promote cellular infiltration, adhesion and proliferation (mesenchymal cells, fibroblasts, osteoblasts, etc.), and favor the differentiation of osteoprogenitor cells that contribute to bone regeneration. In addition, CS-scaffolds allow adequate nutrient transport and promote neovascularization. In order to have better mechanical properties (similarity with bone tissues), a study shows that the use of chitosan scaffolds in combination with chitin-calcium-carbonate-protein complexes can give greater hardness [46]. Another studied application of chitosan is its use as an engineered scaffold in the intestine system for supporting its smooth muscle constructions. Damaged bowel tracts require immediate surgery with possible resection as a result of which the patient generally has malabsorption and malnutrition, so the risk of death remains high [47]. The donor transplant is a technique used but has some limitations such as risk of rejection, lack of availability (long waiting queues), and difficult dimensional matching between donor and recipient [48]. Thus, the challenge is to find an engineering solution that mimics the natural intestine. Natural and synthetic polymers were considered to design a $3 \mathrm{D}$ matrix for seeding cell to promote intestine regeneration. Among these polymers, the use of chitosan in engineering intestinal regeneration is quite known; however, some studies showed that chitosan is well tolerated by the digestive system [49]. Zakhem et al. designed a chitosan/collagen 3D matrix for supporting engineered intestinal muscle constructs able to preserve the normal physiological and morphological (tubular shape with internal lumen) functionality. A volume ratio of $1: 1$ mixture of $2 \%(\mathrm{w} / \mathrm{w})$ chitosan solution dissolved in acetic acid and type I collagen was poured in a custom-made cylindrical mold.

Subsequently, inner lumen was obtained with a tube positioned in the core of the mold; then the system (mold/mixture) was frozen at $-80^{\circ} \mathrm{C}$ and then lyophilized. To obtain the final scaffold, a solution of $\mathrm{NaOH}$ was used to neutralize it, and it was then cross-linked with heparan sulfate, another polysaccharide, with covalent bonds.

\subsubsection{Alginate (ALG)}

Alginic acid is usually called alginate (ALG); it is an anionic hetero-polysaccharide present in the cell walls of algae. Alginates are unbranched, negatively charged polymers composed by variable ratios of $\beta$-D-mannuronate $(\mathrm{M})$ and $\alpha$-L-guluronate $(G)$, where the residues of the blocks $M$ and $G$ are linked by $1-4$ glycosidic bonds [50]. The molecular structure contains blocks of alternating (-MGMG) or consecutive (-GGG- or -MMM-) G or M monomers, and these blocks differ greatly in terms of length and distribution depending on what type and portion of algae are extracted. Generally, the $\mathrm{G}$ amount of algal alginates ranges from $30 \%$ to $70 \%$. The chemical composition and distribution of blocks in the alginate change considerably the properties of alginate; for example, they play a crucial role in their capability of forming ionic gels [51]. At neutral $\mathrm{pH}$, alginate presents a polyanionic nature due to the $3.38 \mathrm{p} K_{\mathrm{a}}$ related to D-mannuronic acid and $3.65 \mathrm{p} K_{\mathrm{a}}$ related to L-gulronic acid. 
The average molecular weights range from 200 to $500 \mathrm{kDa}$. Macro-porous alginate scaffold was used by Yaron Ilan et al. as a biocompatible matrix that protected liver remnants from acute hepatic failure. This scaffold promotes the growth, differentiation, and long-term hepatocellular function of primary hepatocytes in vitro. To evaluate the effect of the use of an alginate scaffold, an experimental group composed of a mouse model, where these scaffolds were implanted after a partial hepatectomy $(\mathrm{PH})$, was compared against untreated mice and against tropes treated with a collagen scaffold. Livers were examined after 3, 6, 24, $48 \mathrm{~h}$, and 6 days following the surgery; alginate scaffolds show a significant animal survival percentage $(60 \%)$ with respect to the nontreated and collagen-treated mice $(10 \%)$. Mice with implanted alginate scaffolds manifested normal and prolonged enzymatic level and more normal levels of determine protein expression level. In conclusion, implanted alginate scaffolds can support liver remnants after extended partial hepatectomy [52]. Buckley et al. developed a porous alginate scaffold with shape-memory properties that recovers its original geometry once hydrated. It can be implanted through minimally invasive approaches.

Once used as intervertebral-disc-like material, this scaffold results cytocompatible and supports cell seeding, penetration, and proliferation. The ability to deliver porous scaffolds using minimally invasive approaches that can potentially promote the regeneration of AF defects provides an exciting new avenue for disc repair [53]. Moreover, in the field of bone tissue engineering, we find alginate as a possible component of biomaterials. In most cases, alginate is used in the form of hydrogel, which has multiple benefits: mechanical stability, satisfying degradability, possibility to repair irregular shape defects due to its capability to cross-link in situ, through minimally invasive surgery $[54,55]$ and its use as drug career for therapeutic controlled release. The crucial point about the use of alginate-based hydrogels for bone regeneration is the formation of hydroxyapatite [56], so most of the studies present in the literature incorporate inside the polymeric matrices salt crystals or other inorganic minerals. For example, the incorporation of hydroxyapatite particles or calcium phosphate in alginate hydrogels improve their mechanical properties, making them more suitable for the bone tissue [57]. However, other biominerals have also been studied for the bone-tissue engineering, and among these, the best candidate seems to be calcium carbonate $\left(\mathrm{CaCO}_{3}\right)$. Calcium carbonates has better biodegradation rates than HAp while maintaining suitable mechanical properties [58]; in addition, it improves the biocompatibility and the osteoinductivity plus promotes the neovascularization in the grafts when implanted in vivo. Diaz-Rodriguez et al. propose the synthesis of a mineralized calcium alginate hydrogel by the addition of calcium carbonate biomineral microparticles. In particular, the $\mathrm{CaCO}_{3}$-alginate hydrogels synthesis consists of: sodium alginate being dissolved on particle suspensions of $\mathrm{CaCO}_{3}$ (obtained from mussels or oyster shells) to obtain a $1 \%$ final alginate concentration. Then the solution was exposed to ultrasound to prevent particle clusterization and transferred in a dialysis membrane; subsequently, it was immersed in $\mathrm{a} \mathrm{CaCl}_{2}$ for $24 \mathrm{~h}$ to promote the cross-linking. At the end, the cross-linked $\mathrm{CaCO}_{3}$-alginate hydrogels were washed in $\mathrm{PBS}$, removed from the 
dialysis membrane, and cut in $1 \mathrm{~mm}$ width discs. Once the hydrogel was obtained, the mechanical properties were evaluated; the experimental data showed that mineralization and the type of alginate used modified tissue mechanical properties in terms of Young's modulus (range of values: 6-12 kPa).

Cytocompatibility, cell adhesion, and ECM mineralization were investigated. $\mathrm{CaCO}_{3}$-alginate hydrogels were characterized by high values of cell viability as expected for biocompatible materials, and the mineralization did not increase cell death or in-cell adhesion (The test was performed with human osteoblasts.). $\mathrm{CaCO}_{3}$ promote ECM mineralization and osteoblast differentiation of mesenchymal stem cells when incorporated within these hydrogels.

\subsubsection{Glycosaminoglycan (GAG)}

Glycosaminoglycans (GAGs) are a class of hetero-polysaccharide that perform some important biological function (as interaction with extracellular matrix protein, enzymes, growth factor ECC) and play a key role in many diseases. GAGs are complex, highly sulfated, polydisperse linear polysaccharides. Depending on the difference of sequencing disaccharide units comprising GAGs, they can be classified into four main groups: chondroitin sulfate/dermatan sulfate, heparin/heparan sulfate, keratan sulfate, and hyaluronic acid (HA). Afterward are shown the principal characteristic and use in tissue regeneration of different GAGs [59].

\subsubsection{Chondroitin sulfate}

Chondroitin sulfate (CS) is a linear, sulfated GAGs consisting of repeating disaccharide units of glucuronic acid and $N$-acetyl-galactosamine usually covalently linked to proteins forming proteoglycans. CS, used with glucosamine, is used as an antiinflammatory to alleviate pain from primary osteoarthritis. CS is an important structural component of cartilage that makes it compression resistant. CS and its isomers have intrinsic properties beyond their physical attributes as a support polymer or as a component that aids in the hydration of a scaffold or wound dressing. Moreover CS presents cell-instructive properties, and growth factor and morphogen-sequestering capability, which equips CS-scaffolds with additional traits to promote cellular proliferation and differentiation conducive to tissue repair processes [60].

\subsubsection{Hyaluronic acid}

Hyaluronic acid (HA) also called hyaluronan is a glycosaminoglycan that is widely present in humans (especially in neural, epithelial, connective tissue). HA is the principal component of the extracellular matrix (ECM) and plays an important physiological role in the human body related to cell proliferation and migration. HA is composed of alternating disaccharide units of D-glucuronic acid and $N$-acetyl-D-glucosamine joined together with $\beta(1,3)$ and $\beta(1,4)$ interglycosidic bonds [61]. In recent years, HA has become widely used in various medical applications (such as dermo-cosmetic, surgery, arthritis treatment, scaffolding, and drug research) due 
its good biodegradability, biocompatibility, nontoxicity, nonimmunogenicity, and noninflammatory properties. Even if HA has these good properties, its application is limited by its poor stability and short biological half-life. To overcome these limits, HA can be chemically modified to improve its properties [62]. HA and its derivatives can be used as drug delivery systems, and they are classified in: nanoparticle, gel and micro-capsule/sphere drug delivery systems, nano-emulsion delivery systems, delivery systems of cationic polymer as carriers for gene drug, polyelectrolyte microcapsule delivery system. An interesting use is in combination with chitosan follows. Myocardial infarction, usually called "heart attack," is a pathologic situation where a part of the heart muscle goes on necrosis following the obstruction (plaques) of one of the coronary arteries. In myocardial infarction, we are in the presence of a decrease in blood flow and a lack of oxygen that could bring patient death.

The first objective of the treatment of myocardial infarction, at the onset of the disease, is to promote the reopening of the occluded coronary artery. In this phase, the time saved between patient arrival and reopening of the vessel results in a gain of cardiac weight before it is irreversibly damaged. The most commonly used technique is the angioplasty that consists of a mechanical expansion of the obstructed vessel with a catheter equipped with an inflatable balloon at the apex. In the absence of angioplasty or the possibility of reaching the coronary arteries with the catheter, there are also drugs that can dissolve the thrombus after being given intravenously (thrombolytic). The drugs are not usable in all patients as they are associated with the possibility of producing very serious bleeding. Another problem is the postmyocardial infarction remodeling. Several materials have been studied for this aim (glutaraldehyde-crosslinked membranes, woven nylon, polytetrafluoroethylene, etc.), but these materials are currently low biocompatible and nonbiodegradable.

Recent experiments on animal models have proved that injection of biomaterials (such as alginate, fibrin, chitosan, collagen, etc.), into the infarcted myocardium could work to improve heart remodeling and the functionality of chronic myocardial infarction [63]. Shoei-Shen Wang et al. proposed chitosan-hyaluronan/silk fibroin (chitosan-HYA/SF) patches for cardiac repair.

The method for producing this chitosan-hyaluronan/silk fibroin patch briefly consists of: boiling silk cocoons in $\mathrm{Na}_{2} \mathrm{CO}_{3}$ to obtain silk fibroin; then it is dissolved in a $\mathrm{LiBr}$ solution and finally dialyzed (silk fibroin aqueous solution was $8 \%$ ). Chitosan were added and dissolved in silk fibroin solution at the concentration of $1.5 \mathrm{wt} \%$ to yield the ratios of chitosan-hyaluronan/silk fibroin as 1:1:10. The silk fibroin-based hybrid micro-particles (fabricated using a spray-drying machine) were pressed using a pressing machine to prepare the patches. The process finished with a cross-linked phase with genipin solution, and the patches were put in distillated water ready for the animal tests [64]. Each patch was examined after 8 weeks of implantation. Rats with scaffold show reduced dilatation of the inner diameter of the left ventricle, increased wall thickness, and improved the fractional shortening of left ventricles of hearts compared with the control group (rats without implants). In the implantation group, they have seen an improved secretion of paracrine factor. In this 
study, the conclusion is that chitosan hyaluronan-silk fibroin patches are a good biomaterial candidate for the cardiac repair following myocardial infraction in the rat model [65].

\subsubsection{Dextran (DeX)}

Dextran is a complex branched glucan where glucans are carbohydrate biomacromolecules made up of d-glucopyranoside units linked by glucosidic bonds through anomeric configurations produced by bacteria, yeasts, fungi, algae, and plants [66]. Dextran is composed of chains of varying lengths where a substantial number of consecutive $\alpha(1,6)$-linkages are in their major chains, usually more than $50 \%$ of the total linkages. Dextran also possesses side-chains, stemming mainly from $\alpha(1,3)$ - and occasionally from $\alpha(1,4)$ - or $\alpha(1,2)$-branched linkages. There is not an exact structure of each type of dextran because it depends on its specific producing microbial strain and, hence, on the specific type of dextransucrase involved [67]. Nontoxicity, biocompatibility, low costs, and the possibility to modify it make dextran widely used and very promising in biomedical and pharmaceutical applications.

The principal uses of dextran are: reducing inflammatory response, decreasing vascular thrombosis, and preventing ischemia. Dextran can act as a mild reactive oxygen species scavenger, reducing excess platelet activation during perfusion in organ transplantation or can be used to replace lost blood in emergency cases in the form of soluble dextran complexes. From the engineering point of view, dextran can be used as coating material to protect and improve biocompatibility or be chemically modified (referred to active hydroxyl group) and used to form scaffold, including spheres, tubules, hydrogels, and nano-structure as nanoparticles [68].

\subsection{Scaffold preparation methods}

The control of the microscale environment is a necessary goal to facilitate cell attachment, growth, and differentiation within polysaccharide-based scaffolds. Moreover, the processing methodology must not adversely affect the material's properties, i.e., their biocompatibility or chemical properties. The most commonly used possibilities presented in Fig. 6.8 are:

- Solvent casting/particulate leaching: scaffold obtained after organic solvent evaporation. This technique allows for an increase in pore interconnectivity. Disadvantages are the necessity to use toxic solvents that could affect biocompatibility and translatability to clinics;

- Fiber bonding: it can guarantee high porosity but no accuracy in microstructure control. This strategy guarantees large surface area, a key point for cell growth; yet they lack structural stability;

- Phase-separation technology: liquid-liquid or solid-liquid separation induced by lowering the solution temperature. The advantage of this is that the activity of the incorporated 


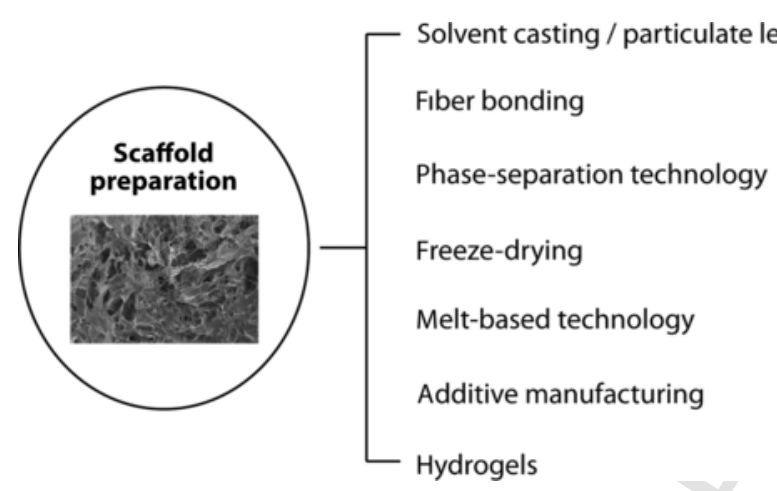

Fig. 6.8 Scaffold preparation methods.

molecules is maintained, while the difficulty of controlling scaffold morphology, together with solvent removal, are the main disadvantages;

- Freeze-drying: it involves a thermally induced phase separation with the removal of the solvent (usually water). The main disadvantages are the low mechanical stability and a pore size distribution in the resulting scaffold in the range of $100 \mu \mathrm{m}$;

- Melt-based technologies: the polymer mold is heated above the glass transition temperature and then immersed in solvents. There are problems in this method regarding nonamorphous polymers, which require a high temperature;

- Solid free-form fabrication (additive manufacturing): these techniques allow high control of microstructure and anisotropy to be obtained; however, the high temperature needed, the presence of solvents, and low mechanical properties should also be considered;

- Hydrogels: suitable because of their ability to retain water and thus they mimick living tissues; they have high biocompatibility, as well as the possibility of precisely controlled release rates of drugs loaded within them. Generally, polysaccharide-based hydrogels are formed to take advantage of electrostatic interactions between polymeric chains (physical gels, Fig. 6.3). The advantages of these systems are the final high injectability, degradability, and possibility to sustain the delivery of drugs into the target tissue. However, the low control in porosity and microstructure, together with the mechanical properties, are the main drawbacks.

\subsection{Summary}

In recent years, the field of transplantation has improved, and tissue engineering represents a promising strategy to increase patient-life expectations and graft survival. This approach is possible thanks to three fundamental components: cells, signals, and scaffolds. Between polymeric scaffolds, polysaccharide-based ones represent a very promising category. Indeed, polysaccharides are able to provide structures extremely similar to living tissues by stimulating a specific cellular response, which sometimes supersedes the advantages of synthetic polymers. Moreover, owing to their similarity with the extracellular matrix, polysaccharides 
may also reduce the stimulation of chronic inflammation or immunological reactions and toxicity, often detected with synthetic polymers.

In summary, polysaccharides present a lot of advantages, but some disadvantages are present as well. In particular, the most important one is represented by the difficulty to be functionalized with chemical groups that can tune degradation rate, mechanical, and chemical properties. Postpolymerization strategies are based on chemoselective orthogonal strategies where functional groups could be linked to polymeric backbone under mild conditions (fine for biomedical applications) and high yields. So in the future, strong attention should be given to these functionalization strategies applied to polysaccharides to improve their performances as scaffolds in tissue engineering.

\section{References}

[1] OPTN: Organ Procurement and Transplantation Network. 2018. Available at https://optn. transplant.hrsa.gov/ [Accessed 3 July 2018].

[2] F.E. Smit, P.M. Dohmen, Cardiovascular tissue engineering: where we come from and where are we now?, Med Sci Monit Basic Res 21 (2015) 1-3.

[3] E.J. Lee, F.K. Kasper, A.G. Mikos, Biomaterials for tissue engineering, Ann Biomed Eng 42 (2014) 323-337.

[4] Y. Wen, J.K. Oh, Recent strategies to develop polysaccharide-based nanomaterials for biomedical applications, Macromol Rapid Commun 35 (2014) 1819-1832.

[5] N. Lin, J. Huang, A. Dufresne, Preparation, properties and applications of polysaccharide nanocrystals in advanced functional nanomaterials: a review, Nanoscale 4 (2012) 3274-3294.

[6] Tissue Engineering and Regenerative Medicine. 2018. Available at https://www.nibib. nih.gov/science-education/science-topics/tissue-engineering-and-regenerative-medicine [Accessed 3 May 2018].

[7] M.Q. Guo, X. Hu, C. Wang, L. Ai, Polysaccharides: structure and solubility, in: Z. Xu (Ed.), Solubility of polysaccharides, InTech, 2017.

[8] J. Liu, S. Willför, C. Xu, A review of bioactive plant polysaccharides: biological activities, functionalization, and biomedical applications, Bioact Carbohydr Diet Fibre 5 (2015) 31-61.

[9] D.W. Hutmacher, J.C. Goh, S.H. Teoh, An introduction to biodegradable materials for tissue engineering applications, Ann Acad Med 30 (2001) 183-191.

[10] T.M. Aminabhavi, Polysaccharide-based hydrogels as biomaterials in drug delivery, J Pharma Care Health Syst 2 (2015) e132.

[11] G. Agoda-Tandjawa, S. Durand, C. Gaillard, C. Garnier, J.L. Doublier, Properties of cellulose/pectins composites: implication for structural and mechanical properties of cell wall, Carbohydr Polym 90 (2012) 1081-1091.

[12] H. Ullah, H.A. Santos, T. Khan, Applications of bacterial cellulose in food, cosmetics and drug delivery, Cellulose 23 (2016) 2291-2314.

[13] I.F. Almeida, T. Pereira, N.H.C.S. Silva, F.P. Gomes, A.J.D. Silvestre, C.S.R. Freire, J.M. Sousa Lobo, P.C. Costa, Bacterial cellulose membranes as drug delivery systems: an in vivo skin compatibility study, Eur J Pharm Biopharm 86 (2014) 332-336.

[14] C.E. Beneke, A.M. Viljoen, J.H. Hamman, Polymeric plant-derived excipients in drug delivery, Molecules 14 (2009) 2602-2620. 
[15] A. Bodin, S. Bharadwaj, S. Wu, P. Gatenholm, A. Atala, Y. Zhang, Tissue-engineered conduit using urine-derived stem cells seeded bacterial cellulose polymer in urinary reconstruction and diversion, Biomaterials 31 (2010) 8889-8901.

[16] S.P. Lin, I. Loira Calvar, J.M. Catchmark, J.R. Liu, A. Demirci, K.C. Cheng, Biosynthesis, production and applications of bacterial cellulose, Cellulose 20 (2013) 2191-2219.

[17] M. Badshah, H. Ullah, S.A. Khan, J.K. Park, T. Khan, Preparation, characterization and in-vitro evaluation of bacterial cellulose matrices for oral drug delivery, Cellulose 24 (2017) 5041-5052.

[18] K.J. Burg, S. Porter, J.F. Kellam, Biomaterial developments for bone tissue engineering, Biomaterials 21 (2000) 2347-2359.

[19] V. Karageorgiou, D. Kaplan, Porosity of 3D biomaterial scaffolds and osteogenesis, Biomaterials 26 (2005) 5474-5491.

[20] M. Zaborowska, A. Bodin, H. Bäckdahl, J. Popp, A. Goldstein, P. Gatenholm, Microporous bacterial cellulose as a potential scaffold for bone regeneration, Acta Biomater 6 (2010) 2540-2547.

[21] J.M. Gutiérrez-Hernández, D.M. Escobar-García, A. Escalante, H. Flores, F.J. González, P. Gatenholm, G. Toriz, In vitro evaluation of osteoblastic cells on bacterial cellulose modified with multi-walled carbon nanotubes as scaffold for bone regeneration, Mater Sci Eng C 75 (2017) 445-453.

[22] S. Saska, L.N. Teixeira, P. Tambasco de Oliveira, A.M. Minarelli Gaspar, S.J. Lima Ribeiro, Y. Messaddeq, R. Marchetto, Bacterial cellulose-collagen nanocomposite for bone tissue engineering, J Mater Chem 22 (2012) 22102-22112.

[23] H. Bäckdahl, M. Esguerra, D. Delbro, B. Risberg, P. Gatenholm, Engineering microporosity in bacterial cellulose scaffolds, J Tissue Eng Regen Med 2 (2008) 320-330.

[24] D. Atila, D. Keskin, A. Tezcaner, Cellulose acetate based 3-dimensional electrospun scaffolds for skin tissue engineering applications, Carbohydr Polym 133 (2015) 251-261.

[25] M. Kim, G. Kim, 3D multi-layered fibrous cellulose structure using an electrohydrodynamic process for tissue engineering, J Colloid Interface Sci 457 (2015) 180-187.

[26] K. Markstedt, A. Mantas, I. Tournier, H. Martínez Ávila, D. Hägg, P. Gatenholm, 3D bioprinting human chondrocytes with nanocellulose-alginate bioink for cartilage tissue engineering applications, Biomacromolecules 16 (2015) 1489-1496.

[27] M.Z. Ahmad, S. Akhter, I. Ahmad, A. Singh, M. Anwar, M. Shamim, F.J. Ahmad, In vitro and in vivo evaluation of Assam bora rice starch-based bioadhesive microsphere as a drug carrier for colon targeting, Expert Opin Drug Deliv 9 (2012) 141-149.

[28] S.K. Nitta, K. Numata, Biopolymer-based nanoparticles for drug/gene delivery and tissue engineering, Int J Mol Sci 14 (2013) 1629-1654.

[29] J. Tian, X. Lin, R. Guan, J.G. Xu, The effects of hydroxyethyl starch on lung capillary permeability in endotoxic rats and possible mechanisms, Anesth Analg 1 (2004) $768-774$.

[30] A.J. Salgado, O.P. Coutinho, R.L. Reis, Novel starch-based scaffolds for bone tissue engineering: cytotoxicity, cell culture, and protein expression, Tissue Eng 10 (2004) 465-474.

[31] V. Patrulea, V. Ostafe, G. Borchard, O. Jordan, Chitosan as a starting material for wound healing applications, Eur J Pharm Biopharm 97 (2015) 417-426. 
[32] S. Senel, S.J. McClure, Potential applications of chitosan in veterinary medicine, Adv Drug Deliv Rev 56 (2004) 1467-1480.

[33] C.N. Costa, V.G. Teixeira, M.C. Delpech, J.V.S. Souza, M.A.S. Costa, Viscometric study of chitosan solutions in acetic acid/sodium acetate and acetic acid/sodium chloride, Carbohydr Polym 133 (2015) 245-250.

[34] M. Rinaudo, Chitin and chitosan: properties and applications, Prog Polym Sci 31 (2006) $603-632$.

[35] H. Kumar, M.N.V. Ravi, R.A.A. Muzzarelli, C. Muzzarelli, H. Sashiwa, A.J. Domb, Chitosan chemistry and pharmaceutical perspectives, Chem Rev 104 (2004) 6017-6084.

[36] J. Kristl, J. Šmid-Korbar, E. Štruc, M. Schara, H. Rupprecht, Hydrocolloids and gels of chitosan as drug carriers, Int J Pharm 99 (1993) 13-19.

[37] J.K. Francis Suh, H.W. Matthew, Application of chitosan-based polysaccharide biomaterials in cartilage tissue engineering: a review, Biomaterials 21 (2000) 2589-2598.

[38] S.V. Madihally, H.W. Matthew, Porous chitosan scaffolds for tissue engineering, Biomaterials 20 (1999) 1133-1142.

[39] S. Dumitriu, Polymeric biomaterials, Marcel Dekker, New York, 2002.

[40] T.M. Freyman, I.V. Yannas, L.J. Gibson, Cellular materials as porous scaffolds for tissue engineering, Progr Mat Sci 46 (2001) 273-282.

[41] N. Boucard, C. Viton, A. Domard, New aspects of the formation of physical hydrogels of chitosan in a hydroalcoholic medium, Biomacromolecules 6 (2005) 3227-3237.

[42] N. Boucard, C. Viton, D. Agay, E. Mari, T. Roger, Y. Chancerelle, A. Domard, The use of physical hydrogels of chitosan for skin regeneration following third-degree burns, Biomaterials 28 (2007) 3478-3488.

[43] P. Kranokpiraksa, D. Pavcnik, H. Kakizawa, B.T. Uchida, M. Jeromel, F.S. Keller, J. Rösch, Hemostatic efficacy of chitosan-based bandage for closure of percutaneous arterial access sites: an experimental study in heparinized sheep model, Radiol Oncol 44 (2010) 86-91.

[44] L. Ma, Collagen/chitosan porous scaffolds with improved biostability for skin tissue engineering, Biomaterials 24 (2003) 4833-4841.

[45] A. Oryan, S. Alidadi, A. Moshiri, N. Maffulli, Bone regenerative medicine: classic options, novel strategies, and future directions, J Orthop Surg Res 9 (2014) 18.

[46] S.N. Danilchenko, O.V. Kalinkevich, M.V. Pogorelov, A.N. Kalinkevich, A.M. Sklyar, T.G. Kalinichenko, V.Y. Ilyashenko, V.V. Starikov, V.I. Bumeyster, V.Z. Sikora, L.F. Sukhodub, Characterization and in vivo evaluation of chitosan-hydroxyapatite bone scaffolds made by one step coprecipitation method, J Biom Mater Res A 96 (2011) 639-647.

[47] T. Schmidt, A. Pfeiffer, N. Hackelsberger, R. Widmer, C. Meisel, H. Kaess, Effect of intestinal resection on human small bowel motility, Gut 38 (1996) 859-863.

[48] G. Brook, Quality of life issues: parenteral nutrition to small bowel transplantation--a review, Nutrition 14 (1998) 813-816.

[49] R.A. Muzzarelli, Chitosan-based dietary foods, Carbohydr Polym 29 (1996) 309-316.

[50] S. Maleki, E. Almaas, S. Zotchev, S. Valla, H. Ertesvåg, Alginate biosynthesis factories in Pseudomonas fluorescens: localization and correlation with alginate production level, Appl Environ Microbiol 82 (2016) 1227-1236.

[51] T. Andersen, P. Auk-Emblem, M. Dornish, 3D cell culture in alginate hydrogels, Microarrays 4 (2015) 133-161. 
[52] E. Shteyer, A. Ben Ya'acov, L. Zolotaryova, A. Sinai, Y. Lichtenstein, O. Pappo, O. Kryukov, T. Elkayam, S. Cohen, Y. Ilan, Reduced liver cell death using an alginate scaffold bandage: a novel approach for liver reconstruction after extended partial hepatectomy, Acta Biomater 10 (2014) 3209-3216.

[53] O. Guillaume, A. Daly, K. Lennon, J. Gansau, S.F. Buckley, C.T. Buckley, Shape-memory porous alginate scaffolds for regeneration of the annulus fibrosus: effect of TGF- $\beta 3$ supplementation and oxygen culture conditions, Acta Biomater 10 (2014) 1985-1995.

[54] V. Guarino, M. Galizia, M. Alvarez-Perez, G. Mensitieri, L. Ambrosio, Improving surface and transport properties of macroporous hydrogels for bone regeneration, J Biomed Mater Res A 103 (2015) 1095-1105.

[55] S. Kim, Y. Kang, A.E. Mercado-Pagán, W.J. Maloney, Y. Yang, In vitro evaluation of photo-crosslinkable chitosan-lactide hydrogels for bone tissue engineering, J Biomed Mater Res B 102 (2014) 1393-1406.

[56] M.M. Stevens, Biomaterials for bone tissue engineering, Mater Today 11 (2008) 18-25.

[57] K. Gkioni, S.C.G. Leeuwenburgh, T.E.L. Douglas, A.G. Mikos, J.A. Jansen, Mineralization of hydrogels for bone regeneration, Tissue Eng Pt B 16 (2010) 577-585.

[58] N. Yang, Q. Zhong, Y. Zhou, S.C. Kundu, J. Yao, Y. Cai, Controlled degradation pattern of hydroxyapatite/calcium carbonate composite microspheres, Microsc Res Tech 79 (2016) 518-524.

[59] F. Zhang, Z. Zhang, R.J. Linhardt, Glycosaminoglycans, In: Handbook of glycomics, Academic Press, San Diego, 2010.

[60] B.L. Farrugia, M.S. Lord, J.M. Whitelock, J. Melrose, Harnessing chondroitin sulphate in composite scaffolds to direct progenitor and stem cell function for tissue repair, Biomater Sci 6 (2018) 947-957.

[61] E.J. Oh, K. Park, K.S. Kim, J. Kim, J.A. Yang, J.H. Kong, M.Y. Lee, A.S. Hoffman, S.K. Hahn, Target specific and long-acting delivery of protein, peptide, and nucleotide therapeutics using hyaluronic acid derivatives, J Control Release 141 (2010) 2-12.

[62] G. Huang, H. Huang, Hyaluronic acid-based biopharmaceutical delivery and tumor-targeted drug delivery system, J Control Release 278 (2018) 122-126.

[63] J. Leor, S. Tuvia, V. Guetta, F. Manczur, D. Castel, U. Willenz, O. Petneházy, N. Landa, M.S. Feinberg, E. Konen, O. Goitein, O. Tsur-Gang, M. Shaul, L. Klapper, S. Cohen, Intracoronary injection of in situ forming alginate hydrogel reverses left ventricular remodeling after myocardial infarction in swine, J Am Coll Cardiol 54 (2009) 1014-1023.

[64] J. Jin, S.I. Jeong, Y.M. Shin, K.S. Lim, H.S. Shin, Y.M. Lee, H.C. Koh, K.S. Kim, Transplantation of mesenchymal stem cells within a poly(lactide-co-epsilon-caprolactone) scaffold improves cardiac function in a rat myocardial infarction model, Eur J Heart Fail 11 (2009) 147-153.

[65] N.H. Chi, M.C. Yang, T.W. Chung, N.K. Chou, S.S. Wang, Cardiac repair using chitosan-hyaluronan/silk fibroin patches in a rat heart model with myocardial infarction, Carbohydr Polym 92 (2013) 591-597.

[66] F.Y. Kagimura, M.A.A. da Cunha, A.M. Barbosa, R.F.H. Dekker, C.R.M. Malfatti, Biological activities of derivatized D-glucans: a review, Int J Biol Macromol 72 (2015) 588-598.

[67] M. Naessens, A. Cerdobbel, W. Soetaert, E.J. Vandamme, Leuconostoc dextransucrase and dextran: production, properties and applications, J Chem Technol Biotechnol 80 (2005) 845-860. 
[68] G. Sun, J.J. Mao, Engineering dextran-based scaffolds for drug delivery and tissue repair, Nanomedicine 7 (2012) 1771-1784. 